\title{
A Novel 111In-Labeled Anti-Prostate-Specific Membrane Antigen Nanobody for Targeted SPECT/CT Imaging of Prostate Cancer
}

\author{
Kristell L.S. Chatalic ${ }^{1,2}$, Joke Veldhoven-Zweistra ${ }^{1}$, Michiel Bolkestein ${ }^{3}$, Sander Hoeben ${ }^{1}$, Gerben A. Koning ${ }^{3}$, \\ Otto C. Boerman ${ }^{4}$, Marion de Jong ${ }^{2}$, and Wytske M. van Weerden ${ }^{1}$ \\ ${ }^{I}$ Department of Urology, Erasmus MC, Rotterdam, The Netherlands; ${ }^{2}$ Departments of Nuclear Medicine and Radiology, Erasmus MC, \\ Rotterdam, The Netherlands; ${ }^{3}$ Laboratory Experimental Surgical Oncology, Department of Surgery, Erasmus MC, Rotterdam, The \\ Netherlands; and ${ }^{4}$ Department of Radiology and Nuclear Medicine, Radboud University Medical Center, Nijmegen, The Netherlands
}

Prostate-specific membrane antigen (PSMA) is overexpressed in prostate cancer $(\mathrm{PCa})$ and a promising target for molecular imaging and therapy. Nanobodies (single-domain antibodies, $\mathrm{V}_{\mathrm{HH}}$ ) are the smallest antibody-based fragments possessing ideal molecular imaging properties, such as high target specificity and rapid background clearance. We developed a novel anti-PSMA Nanobody (JVZ-007) for targeted imaging and therapy of PCa. Here, we report on the application of the ${ }^{111} \mathrm{In}$-radiolabeled Nanobody for SPECT/ CT imaging of PCa. Methods: A Nanobody library was generated by immunization of a llama with 4 human PCa cell lines. Anti-PSMA Nanobodies were captured by biopanning on PSMA-overexpressing cells. JVZ-007 was selected for evaluation as an imaging probe. JVZ-007 was initially produced with a c-myc-hexahistidine (his) tag allowing purification and detection. The c-myc-his tag was subsequently replaced by a single cysteine at the $C$ terminus, allowing site-specific conjugation of chelates for radiolabeling. JVZ-007-cmyc-his was conjugated to 2-(4-isothiocyanatobenzyl)-diethylenetriaminepentaacetic acid ( $p-S C N-D T P A)$ via the lysines, whereas JVZ-007-cys was conjugated to maleimide-DTPA via the C-terminal cysteine. PSMA targeting was analyzed in vitro by cell-binding experiments using flow cytometry, autoradiography, and internalization assays with various PCa cell lines and patient-derived xenografts (PDXs). The targeting properties of radiolabeled Nanobodies were evaluated in vivo in biodistribution and SPECT/CT imaging experiments, using nude mice bearing PSMA-positive PC-310 and PSMA-negative PC-3 tumors. Results: JVZ-007 was successfully conjugated to DTPA for radiolabeling with ${ }^{111} \mathrm{In}$ at room temperature. ${ }^{111}$ In-JVZ007-c-myc-his and ${ }^{111}$ In-JVZ007-cys internalized in LNCaP cells and bound to PSMA-expressing PDXs and, importantly, not to PSMA-negative PDXs and human kidneys. Good tumor targeting and fast blood clearance were observed for ${ }^{111} \mathrm{In}-$ JVZ-007-c-myc-his and ${ }^{111} \mathrm{In}-J V Z-007$-cys. Renal uptake of ${ }^{111} \mathrm{In}-$ JVZ-007-c-myc-his was initially high but was efficiently reduced by coinjection of gelofusine and lysine. The replacement of the c-myc-his tag by the cysteine contributed to a further reduction of renal uptake without loss of targeting. PC-310 tumors were clearly visualized by SPECT/CT with both tracers, with low renal uptake $\left(<4\right.$ percentage injected dose per gram) for ${ }^{111}$ In-JVZ007-cys already at $3 \mathrm{~h}$ after injection. Conclusion: We developed

Received Feb. 27, 2015; revision accepted Apr. 30, 2015.

For correspondence or reprints contact: Kristell Chatalic, Erasmus MC, Department of Nuclear Medicine, Na-620, Postbus 2040, 3000 CA

Rotterdam, The Netherlands.

E-mail: k.chatalic@erasmusmc.nl

Published online May 14, 2015.

COPYRIGHT (c) 2015 by the Society of Nuclear Medicine and Molecular Imaging, Inc. an ${ }^{111}$ In-radiolabeled anti-PSMA Nanobody, showing good tumor targeting, low uptake in nontarget tissues, and low renal retention, allowing excellent SPECT/CT imaging of PCa within a few hours after injection.

Key Words: Nanobody; PSMA; SPECT; ${ }^{111} \mathrm{In}$; prostate cancer

J Nucl Med 2015; 56:1094-1099

DOI: 10.2967/jnumed.115.156729

$\mathbf{P}$ rostate cancer $(\mathrm{PCa})$ is the second leading cause of cancerrelated death among men in the western world. Early detection and accurate staging of $\mathrm{PCa}$ is crucial because the survival rate decreases dramatically when the cancer has spread beyond the prostate (1). Because of the heterogeneity of PCa, and the lack of specificity of conventional imaging techniques, there is currently no universal imaging method approved for detection of early PCa lesions. Prostate-specific membrane antigen (PSMA) is an interesting target for molecular imaging of $\mathrm{PCa}$, as it is overexpressed in $90 \%-100 \%$ of local PCa lesions, as well as on cancerous lymph nodes, and bone metastases (2,3), with some reports suggesting PSMA expression levels are further enhanced in high-grade, metastatic, and castration-resistant PCa $(2,4,5)$. PSMA is also expressed in other tissues including normal prostate epithelium, small intestine, renal tubular cells, and salivary glands, but the expression in these organs is 100-1,000 fold less than in PCa (6).

PSMA, also referred to as glutamate carbopeptidase II (GPCII), $N$-acetyl- $\alpha$-linked acidic dipeptidase I (Naaladase I), or folate hydrolase, is a type II transmembrane glycoprotein exhibiting glutamate carboxypeptidase and folate hydrolase enzymatic activity. The first clinical tracer for imaging PSMA was based on the murine anti-PSMA antibody 7E11, binding to an epitope on the intracellular domain of PSMA. The ${ }^{111}$ In-labeled version of 7E11, ${ }^{111}$ In-capromab, commonly known as ProstaScint (Cytogen Corp.), was approved by the Food and Drug Administration in 1997 for detection of soft-tissue metastases and recurrence of $\mathrm{PCa}$ (7). Its use for staging primary PCa is suboptimal, with an average sensitivity and specificity of $60 \%$ and $70 \%$, respectively (8). ${ }^{111} \mathrm{In}$-capromab was also not reliable for the detection of bone metastases, which are often the initial site of metastasis in advanced PCa (9). After the discovery of 7E11, next-generation monoclonal antibodies (mAbs) binding to the extracellular domain 
of PSMA were developed, including mAb J591. Initially developed for therapeutic purposes, J591 was also evaluated for SPECT imaging in clinical trials, showing characteristics superior to ${ }^{111} \mathrm{In}-$ capromab, revealing most soft-tissue and bony metastases $(10,11)$. Despite improved targeting of J591 and next-generation PSMA $\mathrm{mAb}$, the major disadvantage of the use of antibodies for imaging is the slow clearance from nontarget tissues, often requiring several days between tracer administration and imaging.

An interesting alternative for molecular imaging of PSMA is the development of small-molecule PSMA inhibitors. Because PSMA possesses an enzymatic site in its extracellular domain that cleaves endogenous substrates such as $\mathrm{N}$-acetylaspartylglutamate and poly- $\gamma$-glutamyl folic acid, a series of substrates has been designed. These small-molecule PSMA inhibitors consist of zinc-binding compounds attached to a glutamate moiety. Several radiolabeled PSMA small-molecule inhibitors have been synthetized, starting with phosphonate and phosphate inhibitors, followed by phosphoramidate-, thiol-, and urea-based inhibitors, which are discussed in an extensive review by Mease et al. (12). Some have shown promising results in early clinical studies, such as ${ }^{123} \mathrm{I}-\mathrm{MIP}-1072,{ }^{123} \mathrm{I}-\mathrm{MIP}-1095$ (13), $\mathrm{N}$-[N[(S)-1,3-dicarboxypropyl $]$ carbamoyl $]-4-{ }^{18} \mathrm{~F}$-fluorobenzyl-L-cysteine (14), ${ }^{68} \mathrm{Ga}-\mathrm{HBED}-\mathrm{CC}$ (15), and BAY1075553 (16). These compounds localize rapidly to tumor lesions, including soft-tissue and bone metastases, but also show high uptake in kidneys and salivary glands, attributed to PSMA expression in these organs.

Another approach aiming at circumventing the long circulation time of mAbs is the use of antibody fragments, such as singledomain antibodies $\left(\mathrm{V}_{\mathrm{HH}}\right)$. Nanobodies display attractive features for molecular imaging, including fast nontarget tissue clearance, good tumor penetration capability, and recognition of unique epitopes that are less accessible for mAbs (17). In this study, we describe the development of a Nanobody targeting PSMA and showing good tumor targeting and fast blood clearance, resulting in impressive tumor-to-background ratios within a few hours after injection. The Nanobody was conjugated to a diethylenetriaminepentaacetic acid (DTPA) chelator, allowing facile and stable radiolabeling with ${ }^{111} \mathrm{In}$ at room temperature in a 1-step procedure. The structure of the Nanobody was optimized to minimize renal retention using a novel method for production and labeling of cystagged Nanobodies. We report on the production and radiolabeling of anti-PSMA Nanobodies as well as in vitro and in vivo evaluation in patient-derived PCa xenograft models.

\section{MATERIALS AND METHODS}

\section{Immunization and $\mathbf{V}_{\mathrm{HH}}$ Library Construction}

A llama (Lama glama) was immunized with 4 androgen-responsive human-derived PCa cell lines-LNCaP, PC346C, VCaP, and MDA$\mathrm{PCa}-2 \mathrm{~b}$ - to generate a $\mathrm{V}_{\mathrm{HH}}$ library targeting PCa. The $\mathrm{V}_{\mathrm{HH}}$ library was kindly provided by Dr. Patrick Chames (IBISA). The library construction is described briefly in the supplemental data (supplemental materials are available at http://jnm.snmjournals.org).

\section{Selection and Screening by Phage Display}

A phage display library was produced by infecting the Nanobody library with M13 K07DpIII hyperphages (Progen Biotechnik). PSMAspecific Nanobodies were captured by 3 rounds of biopanning. Each round consisted of a negative selection using wild-type B16 cells followed by a positive selection on B16-PSMA-transfected cells. A selection of single clones was tested for PSMA specificity by enzymelinked immunosorbent assay and flow cytometry. The enzyme-linked immunosorbent assay and flow cytometry protocols are described in the supplemental data.

\section{Nanobody Sequencing, Production, and Purification}

The sequence of JVZ-007 is shown in the supplemental data. Selected clones were produced as c-myc-his-tagged proteins to facilitate purification by affinity chromatography and detection by flow cytometry. Production and purification protocols are described in the supplemental data. Additionally, JVZ-007 Nanobody was produced with a single cysteine at the $\mathrm{C}$ terminus by fusion to SUMO3 ( $\mathrm{Smt} 3$ ubiquitinlike protein) using the pETM11-SUMO3GFP vector (EMBL). Primer design and protein production are described in the supplemental data. The SUMO3 protein was cleaved off by a SUMO-specific protease, Sentrin-specific protease 2 (SenP2, provided by Guy Salvesen, Addgene (18)). Digestion was performed overnight at $4^{\circ} \mathrm{C}$ with a ratio of $1: 10(\mathrm{w} / \mathrm{w})$ of SenP2 to JVZ-007-cys-SUMO3. His-tagged SenP2 and SUMO3 were removed with a HisTrap FF column (GE Healthcare).

\section{Flow Cytometry}

PSMA-specific binding was assessed by flow cytometry on PC346C, LNCaP, and PC-3 cells, using an anti-c-myc mouse primary antibody and a phycoerythrin-conjugated antimouse goat secondary antibody. The flow cytometry protocol is described in the supplemental data.

\section{Conjugation to DTPA and Radiolabeling}

JVZ-007-c- $m y c$-his was incubated with a 5-fold molar excess of p-SCN-Bn-DTPA (Macrocyclics) in $0.1 \mathrm{M}$ sodium carbonate buffer ( $\mathrm{pH} 9.5$ ) for $2.5 \mathrm{~h}$ at room temperature. JVZ-007-cys was reduced with $1 \mathrm{mM}$ 2-mercaptoethylamine- $\mathrm{HCl}$ in phosphate-buffered saline (PBS), $5 \mathrm{mM}$ ethylenediaminetetraacetic acid for $90 \mathrm{~min}$ at $37^{\circ} \mathrm{C}$. Reduced JVZ-007-cys was then incubated with $5 \mathrm{mM}$ maleimide-DTPA for $2 \mathrm{~h}$ at $37^{\circ} \mathrm{C}$. Conjugated Nanobodies were then dialyzed for $3 \mathrm{~d}$ in a SlideA-Lyzer (3.5-kDa cutoff; Life Technologies) against $0.25 \mathrm{M}$ ammonium acetate $\left(\mathrm{NH}_{4} \mathrm{Ac}\right), \mathrm{pH} 5.5$.

Nanobody-DTPA conjugates were labeled with ${ }^{111} \mathrm{InCl}_{3}$ (Covidien) in $20 \mathrm{mM}$ sodium acetate, $\mathrm{pH} 5.0$, for $30 \mathrm{~min}$ at room temperature. Radioprotectants (3.5 mM ascorbic acid, gentisic acid, and methionine) were used to prevent radiolysis. Labeling efficiency was assessed by instant thin-layer chromatography using silica gel-coated paper (Varian Inc.) and $0.1 \mathrm{M}$ citrate buffer, pH 5.0, as the mobile phase. After incubation, an excess of DTPA (final concentration, $0.15 \mathrm{mM}$ ) was added to complex free ${ }^{111} \mathrm{InCl}_{3}$.

\section{Cell Culture, Autoradiography, and Internalization}

Cell lines were purchased from the American Type Culture Collection. B16-PSMA was kindly provided by Marco Colombatti (University of Verona). Cell culture protocols are described in the supplemental data.

Binding of ${ }^{111} \mathrm{In}-\mathrm{JVZ}-007-\mathrm{c}-\mathrm{myc}$-his and ${ }^{111} \mathrm{In}-\mathrm{JVZ}-007$-cys to frozen cryostat section of PDXs and kidneys (mouse/human) was evaluated using autoradiography, as described previously (19). Tissue sections were incubated for $1 \mathrm{~h}$ with ${ }^{111} \mathrm{In}-\mathrm{JVZ}-007-\mathrm{c}-m y c$-his or ${ }^{111} \mathrm{In}-\mathrm{JVZ}-007-c y s$ $\left(10^{-9} \mathrm{M}\right)$. In saturation binding experiments, concentrations ranging from $10^{-6}$ to $10^{-12} \mathrm{M}$ of ${ }^{111} \mathrm{In}-\mathrm{JVZ}-007-\mathrm{c}-m y c$-his were used.

The internalization of ${ }^{111} \mathrm{In}-\mathrm{JVZ}-007-\mathrm{c}-$ $m y c$-his and ${ }^{111} \mathrm{In}-\mathrm{JVZ}-007$-cys was assessed using LNCaP and PC-3 cells. Cells were trypsinized and incubated with ${ }^{111} \mathrm{In}-J V Z-007-c-m y c$-his or ${ }^{111}$ In-JVZ-007-cys $\left(10^{-10} \mathrm{M}\right)$ in RPMI 1640/GlutaMAX (Life Technologies)/20 mM N-(2-hydroxyethyl)piperazine- $N^{\prime}$-(2-ethanesulfonic acid)/1\% bovine serum albumin (pH 7.4) for $90 \mathrm{~min}$ at $37^{\circ} \mathrm{C}$ or $4^{\circ} \mathrm{C}$. JVZ-007-c-myc-his was used for blocking $\left(10^{-6} \mathrm{M}\right)$. After incubation, the cells were centrifuged and washed 2 times with PBS. Cell surface-bound Nanobodies were eluted using a solution of $50 \mathrm{mM}$ glycine/100 mM NaCl (pH 2.8), and cells were washed with PBS. Cells were then lysed in $1 \mathrm{M}$ sodium hydroxide to collect internalized Nanobody. Membrane-bound and internalized fractions were counted in a $\gamma$ counter. Binding was expressed as percentage of added amount of radioactivity per number of cells. 


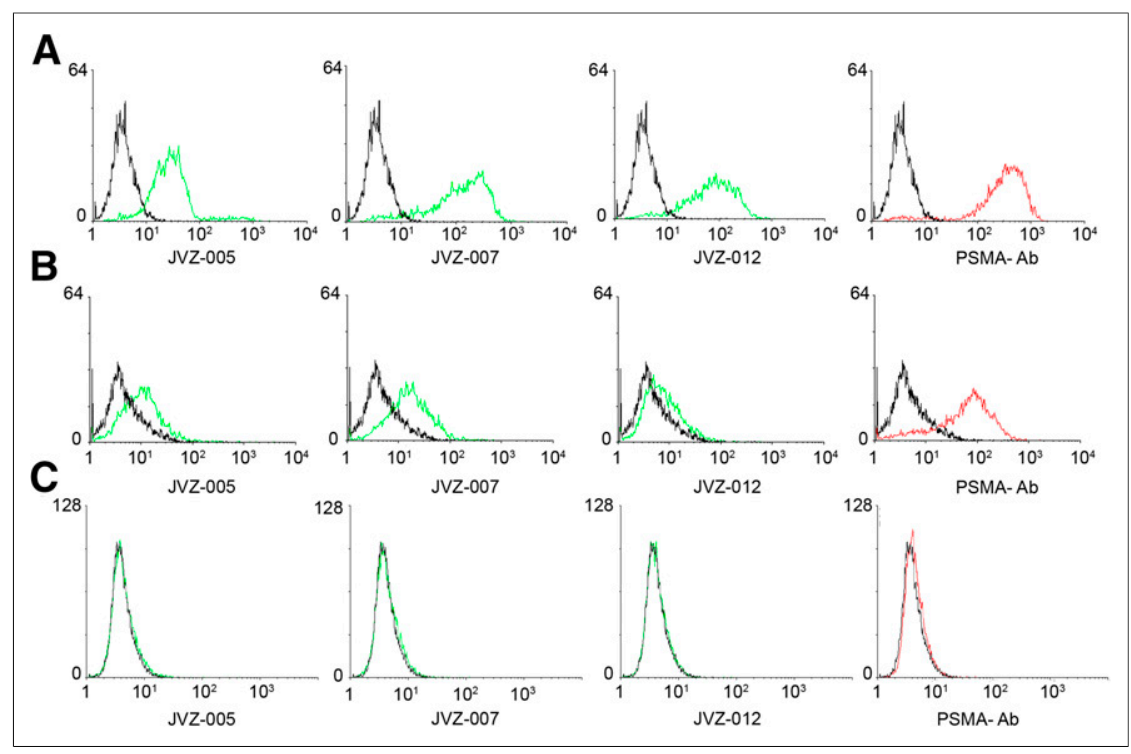

FIGURE 1. Flow cytometry analysis of JVZ-005, JVZ-007, and JVZ-012 on LNCaP (A), PC346C (B), and PC-3 cells (C). Binding was observed on PSMA-positive LNCaP and PC346C cells, whereas no binding was observed on PSMA-negative PC3 cells. Green = in presence of Nanobody; red = in presence of PSMA-antibody (PSMA-Ab); black = control, in absence of Nanobody; $x$-axis $=$ fluorescence intensity; $y$-axis $=$ number of events.

\section{Biodistribution and SPECT/CT Imaging}

Male NMRI $n u / n u$ mice ( 8 wk old) were transplanted with PC-310 tumor fragments near the left shoulder and injected subcutaneously near the right shoulder with PC-3 cells $\left(3 \times 10^{6}\right.$ cells, $200 \mu \mathrm{L}, 66 \%$ RPMI, 33\% Matrigel [BD Bioscience]). Three to $4 \mathrm{wk}$ after inoculation, when tumor size averaged $200 \mathrm{~mm}^{3}$, mice were injected intravenously with radiolabeled Nanobody $(200 \mu \mathrm{L})$. Radiolabeled Nanobody was diluted in PBS containing $0.1 \% \mathrm{v} / \mathrm{w}$ bovine serum albumin. When coinjection of lysine $(20 \mathrm{mg})$ and gelofusine $(4 \mathrm{mg})$ was performed, radiolabeled Nanobody was mixed $1: 1$ with a solution of lysine $(400 \mathrm{mg} / \mathrm{mL})$, and $100 \mu \mathrm{L}$ of this solution were coinjected with $100 \mu \mathrm{L}$ of gelofusine $(40 \mathrm{mg} / \mathrm{mL})$.

Mice were injected with $0.7 \mathrm{MBq}$ of radiolabeled Nanobody (amount ranging from 1 to $100 \mu \mathrm{g}$ ) and euthanized 4 or $24 \mathrm{~h}$ after injection for biodistribution studies. Blood, tumor, and relevant organs and tissues were collected, weighed, and counted in a $\gamma$ counter. The percentage injected dose per gram $(\% \mathrm{ID} / \mathrm{g})$ was determined for each tissue sample.

For SPECT/CT imaging experiments, mice were injected with $30 \mathrm{MBq}$ of ${ }^{111} \mathrm{In}-\mathrm{JVZ} 007$ c-myc-his $(10 \mu \mathrm{g})$ or $15 \mathrm{MBq}$ of ${ }^{111} \mathrm{In}$ JVZ007-cys $(10 \mu \mathrm{g})$. Mice were scanned under isoflurane $/ \mathrm{O}_{2}$ anesthesia at 3 and $24 \mathrm{~h}$ after injection on a small-animal nano-SPECT scanner (Mediso) with heated bed. Scanning, reconstruction, and counting parameters are described in the supplemental data. All animal experiments were approved by the Animal Experiments Committee under the Dutch Experiments on Animal Act and adhered to the European Convention for Protection of Vertebrate Animals used for Experimental Purposes (Directive 86/609/EEC).

\section{Statistical Analysis}

Statistical analyses are described in the supplemental data.

\section{RESULTS}

\section{Generation of Anti-PSMA Nanobodies}

A PCa-specific Nanobody library was generated by immunization of a llama with 4 PCa cell lines (LNCaP, PC346C, VCaP, and MDA-PCa-2b). PSMA-specific Nanobodies were retrieved by biopanning using phage display for 3 positive and negative selection rounds with B16-PSMA and B16 cell lines, respectively. Selection on PSMA-expressing cells was preferred rather than using the recombinant PSMA protein, to increase the chance of capturing Nanobodies binding to an accessible epitope on the extracellular domain of PSMA. Several distinct PSMA-specific Nanobodies were isolated and sequenced (data not shown). Selected clones were produced as c-myc-histagged proteins to facilitate purification by affinity chromatography and detection by flow cytometry. PSMA-specific binding was assessed by flow cytometry on PC-346C, LNCaP, and PC-3 cells. Results of flow cytometry analysis of a few selected Nanobodies are displayed in Figure 1. JVZ-005, JVZ007, and JVZ-012 all bound to PSMAexpressing LNCaP and PC346C, as shown by the increase in the geometric mean of the fluorescence intensity. No binding was observed on the PSMA-negative PC3 cells, showing the specificity of the Nanobodies for PSMA. JVZ-007, showing the highest binding, was selected for further evaluation as the imaging probe. Its sequence is indicated in Supplemental Figure 1. JVZ007-c-myc-his and JVZ-007-cys were successfully produced in bacteria using the pHEN and pETM11-SUMO3GFP expression vectors, respectively.

FIGURE 2. (A) Saturation binding of ${ }^{111} \mathrm{In}-\mathrm{JVZ}-007-\mathrm{C}-\mathrm{myc}$-his on PC-310 frozen sections using autoradiography. (B) Binding of ${ }^{111} \mathrm{In}-J V Z-007-c-m y c-h i s$ and ${ }^{111} \mathrm{In}-J V Z-007$-cys to tumor and kidney frozen sections using autoradiography. High binding is observed on PSMA-expressing tumor sections (PC310, PC346c, LNCaP, PC82, and PC295) whereas no binding is observed on the PSMA-negative tumors (PC3, VCaP, PC133, PC339, and PC324) and kidneys (mouse or human). $\mathrm{B}_{\max }=$ maximum binding; $\mathrm{DLU}=$ digital light unit; $\mathrm{K}_{\mathrm{D}}=$ equilibrium dissociation constant.

\section{Labeling and In Vitro Binding Studies}

JVZ-007-c-myc-his was conjugated to p-SCN-Bn-DTPA via lysine residues, to allow radiolabeling with ${ }^{111}$ In for SPECT imaging of PCa. JVZ-007-cys was site-specifically conjugated with DTPA by reacting it with 


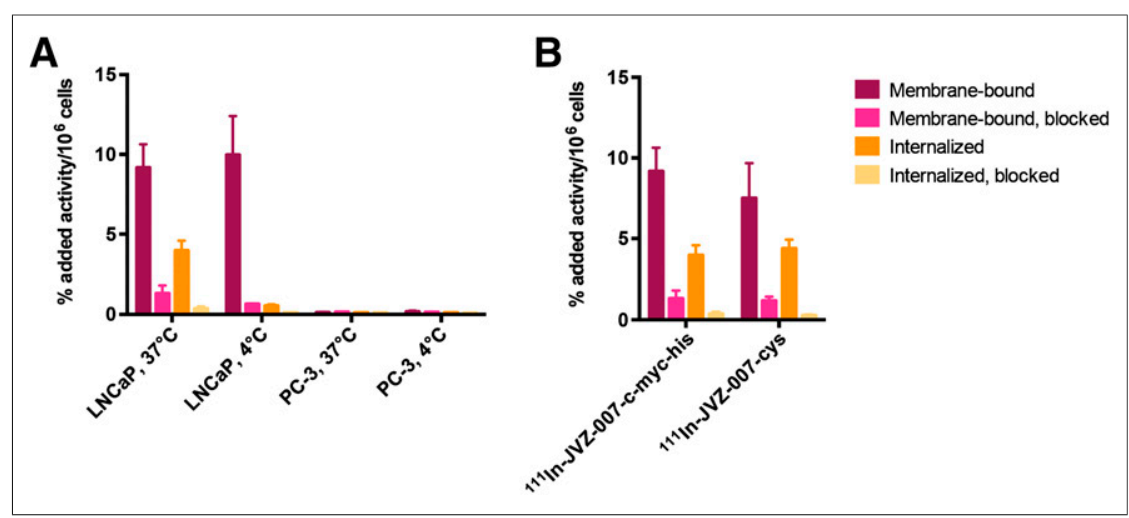

FIGURE 3. (A) Internalization of ${ }^{111} \mathrm{In}-\mathrm{JVZ}-007-\mathrm{c}-m y c-h i s$ in $\mathrm{LNCaP}$ and PC-3 cells, after incubation for $90 \mathrm{~min}$ at $37^{\circ} \mathrm{C}$ and $4^{\circ} \mathrm{C}$. (B) Comparison of internalization of ${ }^{111} \mathrm{In}-\mathrm{JVZ}-007-\mathrm{C}-$ myc-his and ${ }^{111} \mathrm{In}-\mathrm{JVZ}-007$-cys in LNCaP cells after incubation for $90 \mathrm{~min}$ at $37^{\circ} \mathrm{C}$. ${ }^{111} \mathrm{In}-\mathrm{JVZ}$ 007-c-myc-his and ${ }^{111} \mathrm{In}-J \mathrm{VZ}-007$-cys internalized in PSMA-expressing LNCaP cells at $37^{\circ} \mathrm{C}$. tumor sections (PC310, LNCaP, PC82, and PC295), with lower binding to the weakly PSMA-positive PC346c. The absence of binding was observed on the PSMA-negative tumors (PC3, VCaP, PC133, PC339, and PC324). These results are in line with previous PSMA RNA expression data of these PDXs (Affymetrix data not shown). Binding to mouse and human kidneys was low $(<1 \%)$ in comparison with PSMApositive tumors.

Cell binding assays on PSMA-positive LNCaP cells (Fig. 3) showed $30 \%$ and $37 \%$ internalization after $90 \mathrm{~min}$ at $37^{\circ} \mathrm{C}$, for ${ }^{111} \mathrm{In}-J V Z-007-c-m y c$-his and ${ }^{111} \mathrm{In}$ JVZ-007-cys, respectively. Cell binding of both tracers was blocked in the presence of an excess of unlabeled JVZ-007-c-myc-his, confirming target specificity. Internalization of maleimide-DTPA. JVZ-007-cys formed dimers via cysteine bridging, and mild reduction was necessary to free the thiol in the cysteine. ${ }^{111}$ In radiolabeling was performed, with specific activities up to $60 \mathrm{MBq} / \mathrm{nmol}$, whereas the labeling efficiency always exceeded $90 \%$. No release of ${ }^{111} \mathrm{In}$ was observed at $4^{\circ} \mathrm{C}$ up to $72 \mathrm{~h}$ after radiolabeling. Binding to frozen sections of PDXs and kidney using autoradiography is shown in Figure 2 and Supplemental Figure 4. Binding affinity of ${ }^{111}$ In-JVZ-007-c-myc-his was estimated by saturation binding on the PSMA-positive PC-310 tumor, resulting in an equilibrium dissociation constant value of $27.4 \mathrm{nM}$ (14.1-40.7, 95\% confidence interval). ${ }^{111}$ In-JVZ-007-c-myc-his and ${ }^{111}$ In-JVZ-007-cys showed high binding to all PSMA-expressing

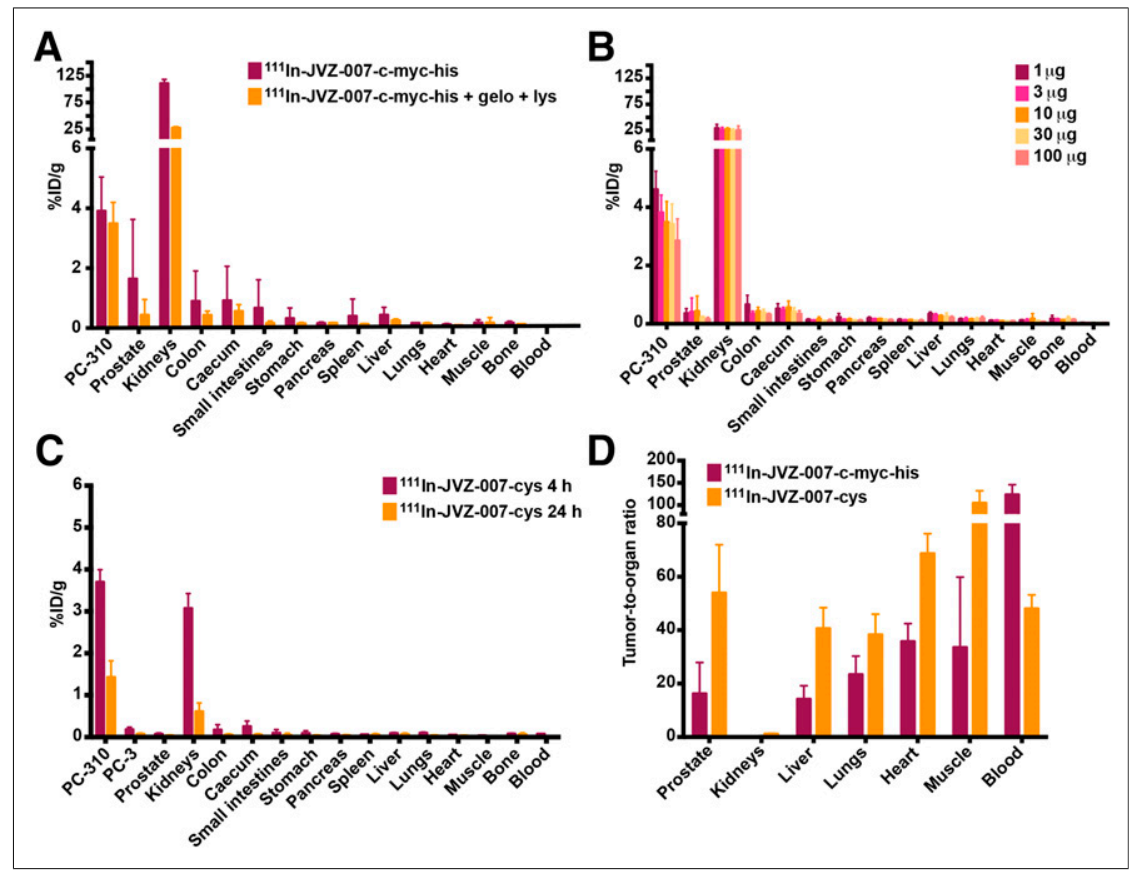

FIGURE 4. Biodistribution studies in mice bearing PC-310 and PC-3 tumors. Effect of coinjection of gelofusine and lysine (A) and dose (B) on biodistribution of ${ }^{111} \mathrm{In}-\mathrm{JVZ}-007-\mathrm{C}-\mathrm{myc}$-his $(10 \mathrm{\mu g})$ at $4 \mathrm{~h}$ after injection (A). (C) Biodistribution at 4 and $24 \mathrm{~h}$ after injection of ${ }^{111} \mathrm{In}-\mathrm{JVZ}-007$-cys $(10 \mu \mathrm{g})$ with coinjection of gelofusine and lysine. (D) Tumor-to-organ ratios at $4 \mathrm{~h}$ after injection of ${ }^{111} \mathrm{In}-J V Z-007-c-m y c-h i s(10 \mu \mathrm{g})$ or ${ }^{111} \mathrm{In}$-JVZ-007-cys $(10 \mu \mathrm{g})$ with coinjection of gelofusine and lysine.
${ }^{111}$ In-JVZ-007-c-myc-his was confirmed by the strongly reduced amount of radioactivity in the internalized fraction $(0.05 \%$ of total binding) observed at $4^{\circ} \mathrm{C}$. Low binding $(<1 \%$ relative to $\mathrm{LNCaP}$ cells) and absence of internalization of ${ }^{111} \mathrm{In}-\mathrm{JVZ}-007-\mathrm{c}-\mathrm{myc}$-his was observed for the PSMA-negative cell line PC-3, confirming Nanobody specificity for PSMA.

\section{Biodistribution and SPECT/CT Imaging}

The PSMA-targeting properties of ${ }^{111} \mathrm{In}-\mathrm{JVZ}-007-\mathrm{c}-\mathrm{myc}$-his and ${ }^{111} \mathrm{In}-J V Z-007$-cys were evaluated further in mice bearing PC-310 and PC-3 tumors. Biodistribution studies are displayed in Figure 4, showing good tumor targeting, with low background intensity except for the kidneys. Four hours after injection, the uptake of ${ }^{111}$ In-JVZ007-c-myc-his in the PC-310 tumor was $3.91 \pm 1.13 \% \mathrm{ID} / \mathrm{g}$. Uptake in the kidneys was $110.89 \pm 7.35 \% \mathrm{ID} / \mathrm{g}$ but could be reduced to $27.77 \pm 2.02 \% \mathrm{ID} / \mathrm{g}$ by coinjection of gelofusine and lysine. Increasing doses of JVZ-007-c-myc-his showed a slight decrease in tumor uptake. Similarly, ${ }^{111}$ In-JVZ-007-cys in combination with gelofusine and lysine showed uptake in PC-310 and PC-3 tumors of $3.70 \pm 0.29$ and $0.18 \pm 0.05 \% \mathrm{ID} / \mathrm{g}$ at $4 \mathrm{~h}$ after injection, respectively. Importantly, kidney uptake was approximately 10 -fold lower than that of JVZ-007-c-myc-his, with values of only $3.13 \pm 0.30$ and $0.61 \pm 0.20 \% \mathrm{ID} / \mathrm{g}$, at 4 and $24 \mathrm{~h}$ after injection, respectively. Moreover, ${ }^{111}$ In-JVZ-007-cys showed higher tumor-to-background ratios at $4 \mathrm{~h}$ after injection than ${ }^{111}$ In-JVZ-007-c-myc-his, with a tumor-to-muscle ratio of $104.8 \pm 27.4$ and tumor-to-kidney ratio of $1.2 \pm 0.1$. The tumor-to-blood ratio was significantly lower for ${ }^{111} \mathrm{In}-\mathrm{JVZ}-007$-cys $(48.1 \pm 5.1)$ than for ${ }^{111}$ In-JVZ-007-c-myc-his.

SPECT/CT images of mice bearing PC310 and PC-3 tumors $3 \mathrm{~h}$ after injection of ${ }^{111}$ In-JVZ-007-c-myc-his and ${ }^{111}$ In-JVZ-007cys are shown in Figure 5. PSMA-expressing 
PC-310 tumor could be clearly visualized with high contrast on the right shoulder, whereas no uptake was observed in PC-3 tumor on the left shoulder. Signal intensity in kidneys after injection of ${ }^{111} \mathrm{In}-$ JVZ-007-c-myc-his was very high but could be efficiently reduced by a coadministration of gelofusine and lysine. Importantly, kidney signal intensity of ${ }^{111} \mathrm{In}$-JVZ-007-cys was markedly lower.

\section{DISCUSSION}

Development of PSMA-based imaging tracers for PCa has increased tremendously in the past few years. After the approval of the PSMA mAb ${ }^{111}$ In-capromab as an imaging agent in 1997 , a panel of novel PSMA-targeted imaging agents was developed, including next-generation antibodies, antibody fragments, aptamers, and PSMA inhibitors (12). PSMA small-molecule inhibitors localize rapidly to tumor lesions, including soft-tissue and bone metastases, but also show a high uptake in the kidneys and salivary

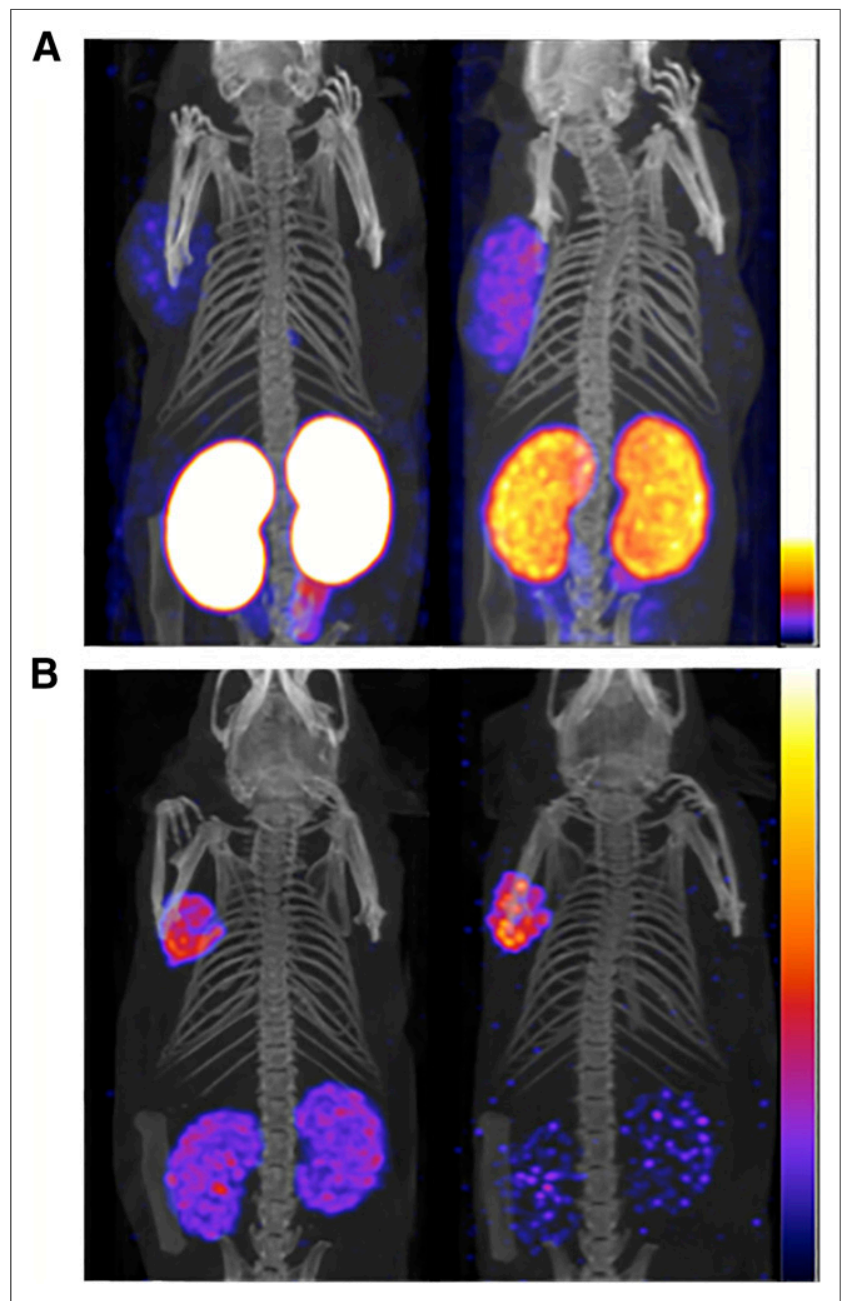

FIGURE 5. SPECT/CT images of mice bearing PC-310 (left shoulder) and PC-3 (right shoulder) tumors. (A) Images acquired at $3 \mathrm{~h}$ after injection of ${ }^{111}$ In-JVZ-007-c-myc-his with coinjection of PBS (left) or gelofusine and lysine (right). (B) Images acquired $3 \mathrm{~h}$ (left) and $24 \mathrm{~h}$ (right) after injection of ${ }^{111} \mathrm{In}-\mathrm{JVZ}-007$-cys with coinjection of gelofusine and lysine. Scale from 0 to $0.2 \mathrm{kBq}(\mathrm{A}) ; 0$ to $0.015 \mathrm{kBq}$ (B, left); 0 to 0.005 $\mathrm{kBq}(\mathrm{B}$, right). PSMA-expressing PC-310 tumor could be clearly visualized with high contrast on left shoulder, whereas no uptake was observed in PC-3 tumor on right shoulder. glands. The high uptake of PSMA inhibitors in these organs was attributed to PSMA expression, although the expression of PSMA in these organs was shown to be 100- to 1,000-fold lower than in PCa (6). This lower PSMA expression in normal organs may suggest that other (receptor-mediated) processes may be involved in tracer uptake in normal organs as well.

In parallel to these developments, alternative strategies have been pursued using smaller variants of mAb, such as antigenbinding fragments $(\mathrm{Fab})$ and $\mathrm{F}\left(\mathrm{ab}^{\prime}\right)_{2}(20)$, and minibodies or diabodies $(21,22)$, aiming to circumvent the long circulation time of $\mathrm{mAb}$. These antibody fragments have shown fast target recognition and rapid blood clearance but also show unspecific accumulation in the liver and kidneys. A more recent approach is the use of Nanobodies $\left(\mathrm{V}_{\mathrm{HH}}\right)$, the smallest antibody-based fragments (12$15 \mathrm{kDa}$ ), offering ideal characteristics for molecular imaging. Because of their small size below the renal threshold for glomerular filtration $(60 \mathrm{kDa})$, they are mainly cleared via the renal pathway. Moreover, it is possible to humanize Nanobodies for clinical translation using the recently described universal humanized Nanobody scaffold technique (23). Nanobodies targeting PSMA were developed, showing moderate tumor targeting, low liver uptake, and high kidney uptake (24). Retention of tracers in the kidneys might be the result of a combination of different factors, including glomerular filtration by the kidneys, PSMA-specific binding, and trapping of metabolites in the lysosomes of renal tubular cells. Tracer retention in the kidneys is not desirable, because it may interfere with visualization of small tumor lesions in the vicinity of the kidneys and especially with staging of PCa. Renal tracer retention also limits the application of the PSMA tracer for radionuclide therapy by inflicting a high dose to the kidneys. We have developed a Nanobody (JVZ-007) that shows good tumor targeting in PSMA-expressing PC-310 PDX tumors. JVZ-007 was initially produced with a c-myc-his-tag and conjugated to p-SCN$\mathrm{Bn}$-DTPA via the lysine residues, to allow radiolabeling with ${ }^{111}$ In for SPECT imaging of PCa. ${ }^{111}$ In-JVZ-007-c-myc-his was internalized in LNCaP cells and showed high binding on all PSMA-expressing PDX sections in vitro, whereas low binding to the PSMA-negative PDX sections was observed and 50-foldlower binding to the human kidney than the PC-310 tumor, indicating the markedly lower expression levels of PSMA in the kidney. ${ }^{111}$ In-JVZ-007-c-myc-his was evaluated further in mice bearing PC-310 tumors, showing good tumor targeting as early as $4 \mathrm{~h}$ after injection, with low background intensity, except for the kidneys. It was recently described that renal retention of an antiepidermal growth factor receptor Nanobody could be reduced by coinjection of gelofusine and lysine (25). Indeed, efficient reduction of renal uptake of our anti-PSMA Nanobody was obtained when gelofusine and lysine were coadministered. These results support that high renal uptake may be due to reabsorption in the renal promixal tubule after glomerular filtration. Moreover, studies from D'Huyvetter et al. (26) have also shown that the his-tag plays a major role in the high retention of radiolabeled Nanobodies in the kidneys. Therefore, we have engineered a new Nanobody construct, based on JVZ-007, in which the c-myc-his tag was removed. In addition, we introduced a cysteine at the $\mathrm{C}$ terminus for site-specific coupling to maleimide-DTPA. For this purpose we used the pETM11-SUMO3GFP expression vector, allowing production of a protein with a C-terminal cysteine and retrieval of the protein by cleavage of SUMO3 by a specific protease. ${ }^{111} \mathrm{In}$ JVZ-007-cys showed similar binding on PSMA-expressing cells and PDXs. More importantly, a further drop in renal uptake to 
$3 \% \mathrm{ID} / \mathrm{g}$ was observed at $4 \mathrm{~h}$ after injection, without loss of tumor targeting. In comparison, these renal uptake values in mice were superior to those found with other PSMA tracers: $100 \% \mathrm{ID} / \mathrm{g}$ for ${ }^{68} \mathrm{Ga}-\mathrm{HBED}-\mathrm{CC}-\mathrm{PSMA}(1 \mathrm{~h})(27)$ and ${ }^{111} \mathrm{In}-\mathrm{D}_{2} \mathrm{~B}-\mathrm{Fab}$ fragments $(4$ h) (20). Kidney uptake of 78 and $36 \% \mathrm{ID} / \mathrm{g}$ was reported for ${ }^{123} \mathrm{I}-$ MIP-1095 and ${ }^{123}$ I-MIP-1072 (4 h), respectively (28). Tumor-toblood and tumor-to-muscle ratios obtained with ${ }^{111}$ In-JVZ-007-cys at $4 \mathrm{~h}$ after injection were superior to those obtained with ${ }^{111} \mathrm{In}$ $\mathrm{D}_{2} \mathrm{~B}-\mathrm{Fab}$ fragments (20) and comparable to those obtained with ${ }^{123} \mathrm{I}$ MIP-1095, in LNCaP xenograft mice (4 h) (28). The novel method described here for production and site-specific labeling of cys-tagged Nanobodies could have a significant impact for the clinical implementation of a wide range of Nanobodies. The site-specific coupling offers a well-defined labeling procedure, while the absence of the c-myc-his-tag limits reabsorption in renal tubular cells. This minimal renal retention also broadens the applicability of this Nanobody to radionuclide therapy.

\section{CONCLUSION}

We developed a specific anti-PSMA Nanobody containing a cysteine for site-specific conjugation to radioactive labels. The ${ }^{111}$ In-radiolabeled anti-PSMA Nanobody shows good tumor targeting and fast blood clearance, allowing SPECT/CT imaging within a few hours after injection. Unlike most radiolabeled small-molecule PSMA inhibitors, anti-PSMA Nanobody JVZ-007 displays a low kidney uptake. These results warrant further evaluation of anti-PSMA Nanobody JVZ-007 for detection and radionuclide therapy of metastatic lesions in PSMAexpressing PCa, for which few treatment options are currently available.

\section{DISCLOSURE}

The costs of publication of this article were defrayed in part by the payment of page charges. Therefore, and solely to indicate this fact, this article is hereby marked "advertisement" in accordance with 18 USC section 1734. This project was funded by the Center for Translational Molecular Medicine (grant 03O-203). Dr. Chatalic ( $\mathrm{PhD}$ student) is employed on a project funded by the Erasmus MC grant "Novel RadioAntagonists for PET/MRI Imaging and Therapy of Prostate Cancer." No other potential conflict of interest relevant to this article was reported.

\section{ACKNOWLEDGMENTS}

We thank Dr. Patrick Chames (Centre de Recherche en Cancérologie de Marseille, France) for the construction of the PCa-specific Nanobody library. We thank Prof. Marco Colombatti and Giulio Fracasso (University of Verona, Italy) for sharing the PSMAtransfected B16 cell line. We thank the EMBL (Heidelberg, Germany) for sharing the pETM11-SUMO3GFP vector. We thank Guy Salvesen for providing the pET28a-SenP2 (catalytic domain) vector. We thank Gerben Franssen for sharing his experience on conjugation of antibodies with chelators. We thank Corrina de Ridder for her assistance during animal experiments.

\section{REFERENCES}

1. Siegel R, Naishadham D, Jemal A. Cancer statistics, 2012. CA Cancer J Clin. 2012;62:10-29.

2. Bostwick DG, Pacelli A, Blute M, Roche P, Murphy GP. Prostate specific membrane antigen expression in prostatic intraepithelial neoplasia and adenocarcinoma: a study of 184 cases. Cancer. 1998;82:2256-2261.

3. Silver DA, Pellicer I, Fair WR, Heston WD, Cordon-Cardo C. Prostate-specific membrane antigen expression in normal and malignant human tissues. Clin Cancer Res. 1997;3:81-85.
4. Wright GL Jr, Haley C, Beckett ML, Schellhammer PF. Expression of prostatespecific membrane antigen in normal, benign, and malignant prostate tissues. Urol Oncol. 1995;1:18-28.

5. Wright GL Jr, Grob BM, Haley C, et al. Upregulation of prostate-specific membrane antigen after androgen-deprivation therapy. Urology. 1996;48:326-334.

6. Sokoloff RL, Norton KC, Gasior CL, Marker KM, Grauer LS. A dual-monoclonal sandwich assay for prostate-specific membrane antigen: levels in tissues, seminal fluid and urine. Prostate. 2000;43:150-157.

7. Ellis RJ, Kaminsky DA, Zhou EH, et al. Ten-year outcomes: the clinical utility of single photon emission computed tomography/computed tomography capromab pendetide (Prostascint) in a cohort diagnosed with localized prostate cancer. Int J Radiat Oncol Biol Phys. 2011;81:29-34.

8. Apolo AB, Pandit-Taskar N, Morris MJ. Novel tracers and their development for the imaging of metastatic prostate cancer. J Nucl Med. 2008;49:2031-2041.

9. Bander NH, Nanus DM, Milowsky MI, Kostakoglu L, Vallabahajosula S, Goldsmith SJ. Targeted systemic therapy of prostate cancer with a monoclonal antibody to prostate-specific membrane antigen. Semin Oncol. 2003;30:667-676.

10. Milowsky MI, Nanus DM, Kostakoglu L, Vallabhajosula S, Goldsmith SJ, Bander NH. Phase I trial of yttrium-90-labeled anti-prostate-specific membrane antigen monoclonal antibody J591 for androgen-independent prostate cancer. J Clin Oncol. 2004;22:2522-2531.

11. Tagawa ST, Beltran H, Vallabhajosula S, et al. Anti-prostate-specific membrane antigen-based radioimmunotherapy for prostate cancer. Cancer. 2010;116:1075-1083.

12. Mease RC, Foss CA, Pomper MG. PET imaging in prostate cancer: focus on prostate-specific membrane antigen. Curr Top Med Chem. 2013;13:951-962.

13. Barrett JA, Coleman RE, Goldsmith SJ, et al. First-in-man evaluation of 2 highaffinity PSMA-avid small molecules for imaging prostate cancer. $\mathrm{J} \mathrm{Nucl} \mathrm{Med}$. 2013;54:380-387.

14. Cho SY, Gage KL, Mease RC, et al. Biodistribution, tumor detection, and radiation dosimetry of ${ }^{18} \mathrm{~F}$-DCFBC, a low-molecular-weight inhibitor of prostatespecific membrane antigen, in patients with metastatic prostate cancer. $\mathrm{J} \mathrm{Nucl}$ Med. 2012;53:1883-1891.

15. Afshar-Oromieh A, Avtzi E, Giesel FL, et al. The diagnostic value of PET/CT imaging with the ${ }^{68} \mathrm{Ga}$-labelled PSMA ligand HBED-CC in the diagnosis of recurrent prostate cancer. Eur J Nucl Med Mol Imaging. 2015;42:197-209.

16. Beheshti M, Kunit T, Haim S, et al. BAY 1075553 PET-CT for staging and restaging prostate cancer patients: comparison with $[\mathrm{F}]$ fluorocholine PET-CT (phase I study). Mol Imaging Biol. 2015;17:424-433.

17. Cortez-Retamozo V, Lauwereys M, Hassanzadeh Gh G, et al. Efficient tumor targeting by single-domain antibody fragments of camels. Int J Cancer. 2002;98:456-462.

18. Mikolajczyk J, Drag M, Bekes M, Cao JT, Ronai Z, Salvesen GS. Small ubiquitinrelated modifier (SUMO)-specific proteases: profiling the specificities and activities of human SENPs. J Biol Chem. 2007;282:26217-26224.

19. Chatalic KL, Franssen GM, van Weerden WM, et al. Preclinical comparison of Al18F- and ${ }^{68} \mathrm{Ga}$-labeled gastrin-releasing peptide receptor antagonists for PET imaging of prostate cancer. J Nucl Med. 2014;55:2050-2056.

20. Lütje S, van Rij CM, Franssen GM, et al. Targeting human prostate cancer with ${ }^{111}$ In-labeled D2B IgG, F(ab')2 and Fab fragments in nude mice with PSMAexpressing xenografts. Contrast Media Mol Imaging. 2015;10:28-36.

21. Viola-Villegas NT, Sevak KK, Carlin SD, et al. Noninvasive imaging of PSMA in prostate tumors with ${ }^{89} \mathrm{Zr}$-labeled huJ591 engineered antibody fragments: the faster alternatives. Mol Pharm. 2014;11:3965-3973.

22. Kampmeier F, Williams JD, Maher J, Mullen GE, Blower PJ. Design and preclinical evaluation of a ${ }^{99 \mathrm{~m}} \mathrm{Tc}$-labelled diabody of mAb J591 for SPECT imaging of prostate-specific membrane antigen (PSMA). EJNMMI Res. 2014;4:13.

23. Vincke C, Loris R, Saerens D, Martinez-Rodriguez S, Muyldermans S, Conrath K. General strategy to humanize a camelid single-domain antibody and identification of a universal humanized nanobody scaffold. J Biol Chem. 2009;284:3273-3284.

24. Evazalipour M, D'Huyvetter M, Tehrani BS, et al. Generation and characterization of nanobodies targeting PSMA for molecular imaging of prostate cancer. Contrast Media Mol Imaging. 2014;9:211-220.

25. Gainkam LO, Caveliers V, Devoogdt N, et al. Localization, mechanism and reduction of renal retention of technetium- $99 \mathrm{~m}$ labeled epidermal growth factor receptor-specific nanobody in mice. Contrast Media Mol Imaging. 2011;6:85-92.

26. D'Huyvetter M, Vincke C, Xavier C, et al. Targeted radionuclide therapy with a ${ }^{177} \mathrm{Lu}$-labeled anti-HER2 nanobody. Theranostics. 2014;4:708-720.

27. Eder M, Schafer M, Bauder-Wust U, et al. ${ }^{68}$ Ga-complex lipophilicity and the targeting property of a urea-based PSMA inhibitor for PET imaging. Bioconjug Chem. 2012;23:688-697.

28. Hillier SM, Maresca KP, Femia FJ, et al. Preclinical evaluation of novel glutamateurea-lysine analogues that target prostate-specific membrane antigen as molecular imaging pharmaceuticals for prostate cancer. Cancer Res. 2009;69: 6932-6940. 